\title{
Lepton flavor violation and seesaw symmetries
}

\author{
D. Aristizabal Sierra
}

\begin{abstract}
When the standard model is extended with right-handed neutrinos the symmetries of the resulting Lagrangian are enlarged with a new global $U(1)_{R}$ Abelian factor. In the context of minimal seesaw models we analyze the implications of a slightly broken $U(1)_{R}$ symmetry on charged lepton flavor violating decays. We find, depending on the $R$-charge assignments, models where charged lepton flavor violating rates can be within measurable ranges. In particular, we show that in the resulting models due to the structure of the light neutrino mass matrix muon flavor violating decays are entirely determined by neutrino data (up to a normalization factor) and can be sizable in a wide right-handed neutrino mass range.
\end{abstract}

Keywords Neutrino mass and mixings - Right handed neutrinos - Decays of leptons

PACS 14.60.Pq $\cdot$ 14.60.St $\cdot 13.35 . \mathrm{Bv} \cdot 13.35 . \mathrm{Dx}$

\section{Introduction}

Apart from demonstrating that neutrinos are massive and have non-vanishing mixing angles among the different generations [1,2, neutrino oscillation experiments have also proved that lepton flavor is not conserved in the neutral lepton sector. Once the standard model is extended to account for neutrino masses - unavoidably - lepton flavor violation (LFV) also takes place in the charged lepton sector. This, however, not necessarily implies that these effects are sizable, so whether these processes can or not have measurable rates depends to a large extent on the details of the corresponding model. Despite this fact, from a general point of view, charged lepton flavor violating processes are expected to have large decay branching fractions as long as the LFV mediators

Diego Aristizabal Sierra

IFPA, Department AGO, Universite de Liege, Bat B5 Sart Tilman B-4000 Liege 1, Belgium. E-mail: daristizabal@ulg.ac.be 
have $\mathcal{O}(\mathrm{TeV})$ masses and their couplings to the standard model leptons are about $\gtrsim 10^{-2}$.

Majorana neutrino masses can be generated in a model independent way by adding the dimension-five effective operator $\mathcal{O}_{5} \sim L L H H$ to the standard model Lagrangian [3]. And in turn the different concrete realizations of this operator constitute a model for neutrino masses 11 Among the tree-level realizations the type-I seesaw is certainly the most popular one [14,15, 16, 17, 18, 19. In this model, light neutrino masses are generated via the exchange of electroweak fermionic singlets (right-handed ( $\mathrm{RH})$ neutrinos for brevity). Consistency with neutrino data then requires either heavy $\mathrm{RH}$ neutrino masses $\left(\mathcal{O}\left(M_{N}\right) \sim \Lambda_{\mathrm{GUT}}\right)$ or tiny Yukawa couplings $\left(\mathcal{O} \sim 10^{-6}\right)$, thus implying negligibly small charged lepton flavor violating effects.

In addition to the standard model gauge symmetry the seesaw Lagrangian features a global Abelian $U(1)_{R}$ symmetry, typically related with phase rotations of the standard model lepton $S U(2)$ singlets, and thus broken by the charged lepton Yukawa couplings. However, relating this symmetry with phase rotations of the $\mathrm{RH}$ leptons fields is not the only possibility, and another approach in which rotations of the left-handed lepton fields and $\mathrm{RH}$ neutrinos are allowed is feasible as well. In that case one is left with (at least) two choices: (i) slightly broken $U(1)_{R} ;$ (ii) $\mathbb{Z}_{n} \subset U(1)_{R}$ invariance of the Lagrangian.

In what follows we will consider possibility $(i)$. In the context of minimal seesaw models (models featuring only $2 \mathrm{RH}$ neutrinos) we will classify the viable scenarios arising from different $R$-charge assignments, that as we already discussed are not anymore limited to the $\mathrm{RH}$ leptons, and identify those models for which charged lepton flavor violating processes have sizable decay branching ratios. For these models we will analyze the $\mu$ flavor violating phenomenology. The results presented here are entirely based on ref. [21.

\section{The models}

Depending on the $R$-charge assignments of the different standard model and RH neutrino fields different models can be constructed. In order to restrict the discussion only to the lepton sector we start by setting $R(H)=0$. Requiring the charged lepton Yukawa couplings to be $U(1)_{R}$ invariant allows to fix $R(e)=$ $R(\ell)$ ( $e, \ell$ being the lepton electroweak singlets and doublets). We are thus left with the lepton doublets and $\mathrm{RH}$ neutrinos $R$-charge assignments. Large lepton flavor violating rates require (at least) the $\mathrm{RH}$ neutrino mass terms to be $U(1)_{R}$ breaking suppressed (the suppression factor denoted by $\epsilon \ll 1$ ), implying $R\left(N_{1,2}\right) \neq 0$ and one of the following three possibilities: (A) $R\left(N_{1}\right)=$ $R\left(N_{2}\right)$; (B) $R\left(N_{1}\right)=-R\left(N_{2}\right)$ or (C) $R\left(N_{1}\right) \neq R\left(N_{2}\right)$. In practice possibilities (A) and (C) turn out to be equivalent as they both lead to models with $N_{1}-N_{2}$ suppressed mixing and therefore to suppressed LFV effects. In contrast in case (B) the $N_{1}-N_{2}$ mixing is maximal and a set of Yukawa couplings can be large

1 Examples range from tree-level up to three-loop induced neutrino mass models $45,6$. \begin{tabular}{l|l|l|l|l|l|l|l|}
\hline & 8 & 9 & 10 & 11 & 12 & 13
\end{tabular} 
provided the $R(\ell)$ charges are chosen appropriately. In that sense models of type (B) are much more interesting as they might yield large LFV effects.

With the purpose of studying the implications for LFV of type B models we fix the $R$-charges as $R\left(N_{1}, \ell_{i}, e_{i}\right)=+1$ and $R\left(N_{2}\right)=-1$. With this charge assignment the seesaw Lagrangian becomes ${ }^{2}$

$$
\mathcal{L}=-\bar{\ell} \boldsymbol{\lambda}_{\mathbf{1}}{ }^{*} N_{1} \tilde{H}-\epsilon_{\lambda} \bar{\ell} \boldsymbol{\lambda}_{\mathbf{2}}{ }^{*} N_{2} \tilde{H}-\frac{1}{2} N_{1}^{T} C M N_{2}-\frac{1}{2} \epsilon_{N} N_{a}^{T} C M_{a a} N_{a}+\text { h.c. }
$$

Here $\tilde{H}=i \sigma_{2} H^{*}, C$ is the charge conjugation operator, $\boldsymbol{\lambda}_{\boldsymbol{a}}^{\dagger}=\left(\lambda_{1 a}^{*}, \lambda_{2 a}^{*}, \lambda_{3 a}^{*}\right)$ with $a=1,2$ (matrices are denoted in bold-face) and $\epsilon_{\lambda, N}$ are dimensionless parameters that slightly break $U(1)_{R}$. Diagonalization of the $\mathrm{RH}$ neutrino mass matrix yields two quasi-degenerate states with masses

$$
M_{N_{1,2}}=M \mp \frac{M_{11}+M_{22}}{2} \epsilon_{N} .
$$

After diagonalization the Yukawa couplings read

$$
\lambda_{k a} \rightarrow-\frac{(i)^{a}}{\sqrt{2}}\left[\lambda_{k 1}+(-1)^{a} \epsilon_{\lambda} \lambda_{k 2}\right], \quad(k=e, \mu, \tau \text { and } a=1,2),
$$

and thus the light neutrino mass matrix is determined to be

$$
\boldsymbol{m}_{\boldsymbol{\nu}}^{\mathrm{eff}}=-\frac{v^{2} \epsilon_{\lambda}}{M}\left|\boldsymbol{\lambda}_{\mathbf{1}}\right||\boldsymbol{\Lambda}|\left(\hat{\boldsymbol{\lambda}}_{\mathbf{1}}^{*} \otimes \hat{\Lambda}^{*}+\hat{\Lambda}^{*} \otimes \hat{\boldsymbol{\lambda}}_{\mathbf{1}}^{*}\right)
$$

with

$$
\hat{\boldsymbol{\Lambda}}^{*}=\hat{\boldsymbol{\lambda}}_{\mathbf{2}}{ }^{*}-\frac{M_{11}+M_{22}}{4 M} \frac{\epsilon_{\lambda}}{\epsilon_{N}} \hat{\boldsymbol{\lambda}}_{\mathbf{1}}{ }^{*} .
$$

Note that the parameter space vectors have been expressed according to $\boldsymbol{\lambda}_{\mathbf{1}}=$ $\left|\boldsymbol{\lambda}_{\mathbf{1}}\right| \hat{\boldsymbol{\lambda}}_{\mathbf{1}}, \boldsymbol{\Lambda}=|\boldsymbol{\Lambda}| \hat{\boldsymbol{\Lambda}}$, where $\hat{\boldsymbol{\lambda}}_{\mathbf{1}}, \hat{\boldsymbol{\Lambda}}$ are unitary vectors along the $\boldsymbol{\lambda}_{\mathbf{1}}, \boldsymbol{\Lambda}$ directions. Due to the structure of the light neutrino matrix the parameter space vectors are - up to normalization factors - completely determined by neutrino mixing angles and masses. For the normal hierarchical spectrum they can be written according to 22]:

$$
\begin{gathered}
\lambda_{\mathbf{1}}=\left|\boldsymbol{\lambda}_{\mathbf{1}}\right| \hat{\boldsymbol{\lambda}}_{\mathbf{1}}=\frac{\left|\boldsymbol{\lambda}_{\mathbf{1}}\right|}{\sqrt{2}}\left(\sqrt{1+\rho} \boldsymbol{U}_{\mathbf{3}}{ }^{*}+\sqrt{1-\rho} \boldsymbol{U}_{\mathbf{2}}{ }^{*}\right), \\
\boldsymbol{\Lambda}=|\boldsymbol{\Lambda}| \hat{\boldsymbol{\Lambda}}=\frac{|\boldsymbol{\Lambda}|}{\sqrt{2}}\left(\sqrt{1+\rho} \boldsymbol{U}_{\mathbf{3}}{ }^{*}-\sqrt{1-\rho} \boldsymbol{U}_{\mathbf{2}}{ }^{*}\right)
\end{gathered}
$$

where the $\boldsymbol{U}_{\boldsymbol{i}}$ 's correspond to the columns of the leptonic mixing matrix and

$$
\rho=\frac{\sqrt{1+r}-\sqrt{r}}{\sqrt{1+r}+\sqrt{r}}, \quad r=\frac{m_{\nu_{2}}^{2}}{m_{\nu_{3}}^{2}-m_{\nu_{2}}^{2}} .
$$

2 Phenomenologically these models are similar to models where lepton number is slightly broken (see e.g. references [22, 23 24 $24,25 \quad 26,27 \quad 28$ ) 


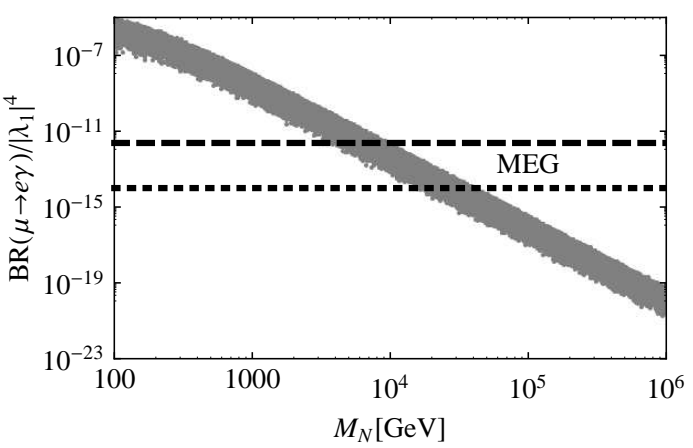

Fig. 1 Decay branching ratio $\operatorname{BR}(\mu \rightarrow e \gamma)$ normalized to $\left|\boldsymbol{\lambda}_{\mathbf{1}}\right|^{4}$ for the normal light neutrino mass spectrum as a function of the common RH neutrino mass. The upper horizontal dashed line indicates the current experimental upper limit from the MEG experiment 34, whereas the lower dotted one marks prospective future experimental sensitivities 30 .

\section{Charged lepton flavor violating decays}

Currently the most competitive bounds on charged lepton flavor violating processes are placed for $\mu$ decays, being $\mu \rightarrow e \gamma, \mu \rightarrow 3 e$ and $\mu-e$ conversion in nuclei the ones with the most stringent upper limits [29. In addition it is for these processes that the most tight bounds are expected in near-future experimental proposals: MEG [30, Muзe 31] PRISM/PRIME 32. So henceforth we will focus on $\mu$ decays, in particular on the reactions $\mu \rightarrow e \gamma$ and $\mu \rightarrow 3 e$ (for $\mu-e$ conversion in nuclei see ref. 21]).

\section{$3.1 \mu \rightarrow e \gamma$ process}

In the limit $M_{W} / M \ll 1$ the decay branching ratio for this decay can be written as 33

$$
\mathrm{BR}(\mu \rightarrow e \gamma) \simeq \frac{\alpha}{1024 \pi^{4}} \frac{m_{\mu}^{5}}{M^{4}} \frac{\left|\lambda_{\mathbf{1}}\right|^{4}}{\Gamma_{\text {Tot }}^{\mu}}\left|\hat{\lambda}_{21} \hat{\lambda}_{11}^{*}\right|^{2}
$$

Thus showing that apart from the parameters $M$ and $\left|\boldsymbol{\lambda}_{\mathbf{1}}\right|$ this branching fraction is entirely determined by low-energy data (see eqs. (6), (7) and (8)). Figure 11 shows the results obtained from the full formula involving the complete one-loop function (see ref. 21 for details) and by randomly generating the low-energy observables in their $2 \sigma$ allowed range [1,2] (normal hierarchical mass spectrum), the parameters $\left|\boldsymbol{\lambda}_{\mathbf{1}}\right|$ and $M$ in the intervals $\left[10^{-5}, 1\right]$ and $\left[10^{2}, 10^{6}\right] \mathrm{GeV}$ and the $N_{1,2}$ mass splittings in the range $\left[10^{-8}, 10^{-6}\right] \mathrm{GeV}$. As can be realized from eqs. (6), (7), (8) and (9) the width of the band is due to neutrino data uncertainties.

From fig. 1 it can be seen that $\operatorname{BR}(\mu \rightarrow e \gamma)$ can reach the current experimental upper bound 34 as long as $M_{N}<0.1 \mathrm{TeV}, 1 \mathrm{TeV}, 10 \mathrm{TeV}$ provided $\left|\boldsymbol{\lambda}_{\mathbf{1}}\right| \gtrsim 2 \times 10^{-2}, 10^{-1}, 1$, respectively. 


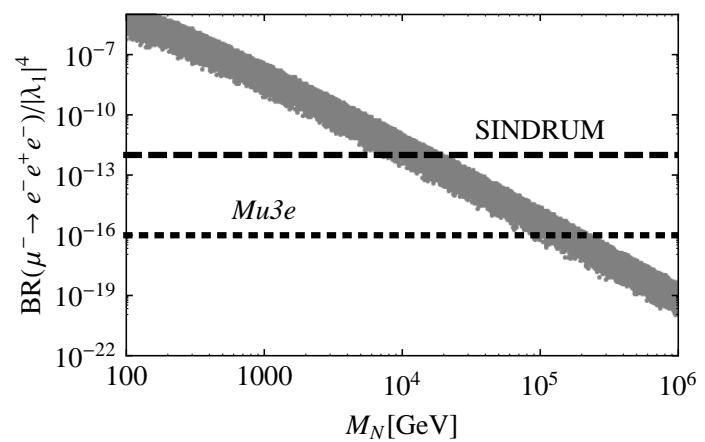

Fig. 2 Decay branching ratio $\operatorname{BR}\left(\mu^{-} \rightarrow e^{-} e^{+} e^{-}\right)$normalized to $\left|\boldsymbol{\lambda}_{\mathbf{1}}\right|^{4}$ for normal light neutrino mass spectrum as a function of the common RH neutrino mass. The upper horizontal dashed line indicates the current bound on the $\mu^{-} \rightarrow e^{+} e^{-} e^{-}$rate placed by the SINDRUM experiment 35, whereas the lower dotted one illustrates prospective future experimental sensitivities of the Muзe experiment [31.

\section{$3.2 \mu \rightarrow 3 e$ process}

We now turn to the discussion of the $\mu \rightarrow 3 e$ process. This decay involves dipole contributions, $\gamma$ and $Z$ penguins as well as box diagrams [33], so a simple approximate formula as in the previous case does not exist. Following the same numerical procedure than in the $\mu \rightarrow e \gamma$ case we calculate the corresponding decay branching ratio. Fig. 2 shows the result for the branching fraction normalized to $\left|\boldsymbol{\lambda}_{\mathbf{1}}\right|^{4}$ for the normal hierarchical mass spectrum.

It can be seen that $\operatorname{BR}(\mu \rightarrow 3 e)$ can exceed the experimental upper limit for $\mathrm{RH}$ neutrino masses $M_{N}<0.1 \mathrm{TeV}, 1 \mathrm{TeV}, 10 \mathrm{TeV}$ provided $\left|\boldsymbol{\lambda}_{\mathbf{1}}\right| \gtrsim$ $2 \times 10^{-2}, 10^{-1}, 1$, respectively, very similar to the $\mu \rightarrow$ e $\gamma$ case. Mainly due to the sensitivities of the planned future experiments $\left(10^{-16}-10^{-15}\right)$ this decay has the potential to probe considerably larger values of the $\mathrm{RH}$ neutrino masses (compared with $\mu \rightarrow e \gamma$ ), reaching $\mathrm{RH}$ neutrino mass scales in excess of $\mathcal{O}\left(10^{5} \mathrm{GeV}\right)$ for $\left|\lambda_{\mathbf{1}}\right| \sim 1$. Finally we note that due to the strong $\left|\boldsymbol{\lambda}_{\mathbf{1}}\right|$ dependence, values of $\left|\boldsymbol{\lambda}_{\mathbf{1}}\right|$ below $10^{-3}$ are not expected to yield observable rates at near future experimental facilities even for $\mathrm{RH}$ neutrino masses of the order $100 \mathrm{GeV}$.

\section{Conclusions}

We studied the implications of the seesaw global Abelian $U(1)_{R}$ symmetry on lepton flavor violation, in the context of minimal seesaw models. We showed that depending on the $R$-charge assignments - generically - two type of models can be identified. A first class where the mechanism that suppresses the light neutrino masses propagates to the lepton flavor violating observables, thus implying negligibly small LFV effects. A second class in which the mechanism "decouples" yielding in that way sizable rates for lepton flavor violating $\mu$ 
decays. We discussed $\mu \rightarrow e \gamma$ and $\mu \rightarrow 3 e$ and showed that these processes might have decay branching fractions within the reach of current or near-future experiments.

Acknowledgements I want to thank Audrey Degee and Jernej F. Kamenik for the fruitful collaboration that led to the paper on which this article is based.

\section{References}

1. T. Schwetz, M. Tortola and J. W. F. Valle, "Where we are on $\theta_{13}$ : addendum to 'Global neutrino data and recent reactor fluxes: status of three-flavour oscillation parameters' ", New J. Phys. 13, 109401 (2011) arXiv:1108.1376 [hep-ph]];

2. M. C. Gonzalez-Garcia, M. Maltoni and J. Salvado, "Updated global fit to three neutrino mixing: status of the hints of $\theta_{13}>0$ ", JHEP 1004, 056 (2010) arXiv:1001.4524 [hep-ph]].

3. S. Weinberg, Phys. Rev. D 22, 1694 (1980).

4. A. Zee, "A Theory of Lepton Number Violation, Neutrino Majorana Mass, and Oscillation", Phys. Lett. B93, 389 (1980).

5. D. Aristizabal Sierra and D. Restrepo, "Leptonic Charged Higgs Decays in the Zee Model," JHEP 0608, 036 (2006) hep-ph/0604012.

6. A. Zee, "Quantum Numbers Of Majorana Neutrino Masses", Nucl. Phys. B 264, 99 (1986).

7. K. S. Babu, "Model of 'Calculable' Majorana Neutrino Masses", Phys. Lett. B 203, 132 (1988)

8. K. S. Babu and C. Macesanu, Two-loop neutrino mass generation and its experimental consequences, Phys. Rev. D67, 073010 (2003) hep-ph/0212058.

9. D. Aristizabal Sierra, M. Hirsch, "Experimental tests for the Babu-Zee two-loop model of Majorana neutrino masses", JHEP 0612, 052 (2006). hep-ph/0609307.

10. M. Nebot, J. F. Oliver, D. Palao, A. Santamaria, "Prospects for the Zee-Babu Model at the CERN LHC and low energy experiments", Phys. Rev. D77, 093013 (2008). arXiv:0711.0483 [hep-ph]].

11. D. Aristizabal Sierra, M. Hirsch, S. G. Kovalenko, "Leptoquarks: Neutrino masses and accelerator phenomenology", Phys. Rev. D77, 055011 (2008). arXiv:0710.5699 [hep-ph]].

12. P. Fileviez Perez, M. B. Wise, "On the Origin of Neutrino Masses", Phys. Rev. D80, 053006 (2009). arXiv:0906.2950 [hep-ph]].

13. K. S. Babu, J. Julio, "Two-Loop Neutrino Mass Generation through Leptoquarks", Nucl. Phys. B841, 130-156 (2010). arXiv:1006.1092 [hep-ph]].

14. P. Minkowski, " $\mu \rightarrow e \gamma$ at a rate of one out of 1-Billion muon decays?", Phys. Lett. B 67, 421 (1977).

15. R. N. Mohapatra and G. Senjanovic, "Neutrino Mass and Spontaneous Parity Violation", Phys. Rev. Lett. 44, 912 (1980).

16. T. Yanagida, "Horizontal Symmetry And Masses Of Neutrinos", Conf. Proc. C 7902131, 95 (1979).

17. M. Gell-Mann, P. Ramond and R. Slansky, "Complex Spinors And Unified Theories", Conf. Proc. C 790927, 315 (1979).

18. S. L. Glashow, "The Future Of Elementary Particle Physics", NATO Adv. Study Inst. Ser. B Phys. 59, 687 (1980).

19. J. Schechter and J. W. F. Valle, "Neutrino Masses in $S U(2) \times U(1)$ Theories", Phys. Rev. D 22, 2227 (1980).

20. R. Alonso, G. Isidori, L. Merlo, L. A. Munoz and E. Nardi, JHEP 1106, 037 (2011) arXiv:1103.5461 [hep-ph]].

21. D. Aristizabal Sierra, A. Degee and J. F. Kamenik, JHEP 1207, 135 (2012) arXiv:1205.5547 [hep-ph]].

22. M. B. Gavela, T. Hambye, D. Hernandez and P. Hernandez, "Minimal Flavour Seesaw Models", JHEP 0909, 038 (2009) arXiv:0906.1461 [hep-ph]]. 
23. R. N. Mohapatra and J. W. F. Valle, "Neutrino Mass and Baryon Number Nonconservation in Superstring Models", Phys. Rev. D 34, 1642 (1986);

24. G. C. Branco, W. Grimus and L. Lavoura, "The Seesaw Mechanism In The Presence Of A Conserved Lepton Number", Nucl. Phys. B 312, 492 (1989);

25. A. Abada, C. Biggio, F. Bonnet, M. B. Gavela and T. Hambye, "Low energy effects of neutrino masses", JHEP 0712, 061 (2007) arXiv:0707.4058 [hep-ph]].

26. P. -H. Gu, M. Hirsch, U. Sarkar and J. W. F. Valle, "Neutrino masses, leptogenesis and dark matter in hybrid seesaw", Phys. Rev. D 79, 033010 (2009) arXiv:0811.0953 [hep-ph]].

27. D. Ibanez, S. Morisi and J. W. F. Valle, "Inverse tri-bimaximal type-III seesaw and lepton flavor violation", Phys. Rev. D 80, 053015 (2009) arXiv:0907.3109 [hep-ph]]

28. D. V. Forero, S. Morisi, M. Tortola and J. W. F. Valle, "Lepton flavor violation and non-unitary lepton mixing in low-scale type-I seesaw", JHEP 1109, 142 (2011) arXiv:1107.6009 [hep-ph]]

29. K. Nakamura et al. [Particle Data Group Collaboration], "Review of particle physics", J. Phys. G G 37, 075021 (2010).

30. http://meg.icepp.s.u-tokyo.ac.jp/docs/prop_psi/proposal.pdf

31. http://www.physi.uni-heidelberg.de/Forschung/he/mu3e/documents/LOI_Mu3e_PSI.pdf

32. C. Ankenbrandt et al., "Using the Fermilab proton source for a muon to electron conversion experiment", arXiv:physics/0611124

33. A. Ilakovac and A. Pilaftsis, "Flavor violating charged lepton decays in seesaw-type models", Nucl. Phys. B 437, 491 (1995) hep-ph/9403398.

34. J. Adam et al. [MEG Collaboration], "New limit on the lepton-flavour violating decay $\mu^{+} \rightarrow e^{+} \gamma^{\prime}$, Phys. Rev. Lett. 107, 171801 (2011) arXiv:1107.5547 [hep-ex]].

35. U. Bellgardt et al. [SINDRUM Collaboration], "Search for the Decay $\mu^{+} \rightarrow e^{+} e^{+} e^{-}$", Nucl. Phys. B 299, 1 (1988). 